\title{
An astro-comb calibrated solar telescope to search for the radial velocity signature of Venus
}

\section{Citation}

Phillips,D.F., A. G. Glenday, X. Dumusque, N. Buchschacher, A. C. Cameron, M. Cecconi, D. Charbonneau, R. Cosentino, A. Ghedina, R. Haywood, D. W. Latham, C-H. Li, M. Lodi, C. Lovis, E. Molinari, F. Pepe, D. Sasselov, A. Szentgyorgyi, S. Udry, and R. L. Walsworth. 2016. An astrocomb calibrated solar telescope to search for the radial velocity signature of Venus. Advances in Optical and Mechanical Technologies for Telescopes and Instrumentation II, edited by Ramón Navarro, James H. Burge, Proc. of SPIE Vol. 9912: 99126Z. doi: 10.1117/12.2232452

\section{Published Version}

doi: $10.1117 / 12.2232452$

\section{Permanent link}

http://nrs.harvard.edu/urn-3:HUL.InstRepos:29374076

\section{Terms of Use}

This article was downloaded from Harvard University's DASH repository, and is made available under the terms and conditions applicable to Other Posted Material, as set forth at http:// nrs.harvard.edu/urn-3:HUL.InstRepos:dash.current.terms-of-use\#LAA

\section{Share Your Story}

The Harvard community has made this article openly available. Please share how this access benefits you. Submit a story. 


\title{
An astro-comb calibrated solar telescope to search for the radial velocity signature of Venus
}

\author{
David F. Phillips ${ }^{\mathrm{a}}$, Alex G. Glenday ${ }^{\mathrm{b}}$, Xavier Dumusque ${ }^{\mathrm{c}}$, Nicolas Buchschacher ${ }^{\mathrm{c}}$, Andrew \\ Collier Cameron $^{\mathrm{d}}$, Massimo Cecconi ${ }^{\mathrm{e}}$, David Charbonneau ${ }^{\mathrm{a}}$, Rosario Cosentino ${ }^{\mathrm{e}}$, Adriano \\ Ghedina $^{\mathrm{e}}$, Raphä̈lle Haywood ${ }^{\mathrm{a}}$, David W. Latham ${ }^{\mathrm{a}}$, Chih-Hao Li ${ }^{\mathrm{f}}$, Marcello Lodi ${ }^{\mathrm{e}}$, Christophe \\ Lovis $^{\mathrm{c}}$, Emilio Molinarie,, Francesco Pepe ${ }^{\mathrm{c}}$, Dimitar Sasselov ${ }^{\mathrm{a}}$, Andrew Szentgyorgyia \\ Stephane Udry ${ }^{\mathrm{c}}$, and Ronald L. Walsworth \\ ${ }^{a}$ Harvard-Smithsonian Center for Astrophysics, Cambridge, MA USA 02138. \\ ${ }^{\mathrm{b}}$ Komodo Health Inc., New York NY USA. \\ ${ }^{\mathrm{C}}$ Observatoire de Genéve, 51 Ch. des Maillettes, 1290 Sauverny, Switzerland. \\ ${ }^{\mathrm{d} S U P A}$, School of Physics and Astronomy, University of St Andrews, St Andrews KY16 9SS, \\ UK. \\ e'NAF-Fundacion Galileo Galilei, Rambla Jose Ana Fernandez Perez 7, E-38712 Brena Baja, \\ Spain. \\ ${ }^{\mathrm{f}}$ AOSense, Inc., Sunnyvale CA USA 94085. \\ ${ }^{g}$ INAF-IASF Milano, via Bassini 15, I-20133, Milano, Italy.
}

June 16, 2016

\begin{abstract}
We recently demonstrated sub-m/s sensitivity in measuring the radial velocity (RV) between the Earth and Sun using a simple solar telescope feeding the HARPS-N spectrograph at the Italian National Telescope, which is calibrated with a green astro-comb. We are using the solar telescope to characterize the effects of stellar (solar) RV jitter due to activity on the solar surface with the goal of detecting the solar RV signal from Venus, thereby demonstrating the sensitivity of these instruments to detect true Earth-twin exoplanets.
\end{abstract}

\section{A SOLAR TELESCOPE ON HARPS-N}

Detecting terrestrial exoplanets in the habitable zones of Sun-like stars using the radial velocity (RV) method is currently challenged by perturbations induced by stellar surface inhomogeneities including spots, plages and granules leading to jitter in the measured stellar RV. Such stellar jitter is not well understood and is difficult to study in stars other than the Sun because their surfaces are unresolved. We are therefore observing the RV of the Sun by using a small, low-cost solar telescope and the HARPS-N spectrograph, calibrated with a green astrocomb, a laser frequency comb optimized for calibrating high-resolution astrophysical spectrographs. ${ }^{1}$ This simple solar telescope, consisting of a lens feeding an integrating sphere and coupled to the HARPS-N spectrograph via an optical fiber, now operates automatically, every clear day. We characterized this telescope both in the lab and on-sky to insure: (i) sufficient scrambling by the integrating sphere; (ii) insensitivity to guiding; and (iii) consistent throughput, independent of acceptance angle such that light from the Sun fed to HARPS-N mimics the light of an unresolved star. We recently demonstrated sub-m/s sensitivity in measuring the Earth-Sun RV with this instrument. Daily observations of the Sun will allow us to characterize the sources of stellar RV jitter; and with the help of solar satellites such as the Solar Dynamics Observatory (SDO), we will characterize the correlation between observed RV and detailed imaging of the solar photosphere. We plan to use these tools to mitigate the effects of stellar jitter with the goal of the detection of Venus from its solar RV signature, thus showing the potential of the RV technique to detect true Earth-twin exoplanets.

Further author information: (Send correspondence to D.F.P.)

E-mail: dphil@cfa.harvard.edu

Advances in Optical and Mechanical Technologies for Telescopes and Instrumentation II, edited by Ramón Navarro, James H. Burge, Proc. of SPIE Vol. 9912, $99126 Z$

(C) 2016 SPIE · CCC code: 0277-786X/16/\$18 - doi: 10.1117/12.2232452

Proc. of SPIE Vol. 9912 99126Z-1 


\section{HARPS-N}

HARPS-N is a fiber-fed, cross-dispersed echelle spectrograph, optimized for radial velocity exoplanet detection operating at the $3.6 \mathrm{~m}$ Telescopio Nazionale Galileo (TNG) at the Observatorio del Roque de los Muchachos, Spain. $^{2}$ HARPS-N provides a spectral range from $390 \mathrm{~nm}$ to $690 \mathrm{~nm}$ with a resolving power of $R=115000$. Two fibers, science and reference, feed the spectrograph with calibration and stellar light. The fiber entrance is re-imaged by the spectrograph optics onto a $4 \mathrm{k} 4 \mathrm{k}$ CCD, where echelle spectra of 69 orders are formed for each fiber. The spectrograph is mounted on a nickel plated stainless steel mount and contains no moving parts. Furthermore, in order to avoid spectral drifts due to temperature and air pressure variations, it is accurately controlled in pressure and temperature.

With an achieved precision of better than 1 meter per second, HARPS-N has revealed and characterized rocky planets in a variety of orbits including: Kepler- $78,{ }^{3}$ a $1.86 m_{\oplus}$ mass planet with a radius of $1.16 R_{\oplus}$ in an 8.5 hour orbit; HD $219134 \mathrm{~b},{ }^{4}$ a nearby $(6.5 \mathrm{pc})$ transiting planet found as part of the HARPS-N Rocky Planet Search (RPS) with a mass of $4.5 m_{\oplus}$, a radius of $1.6 R_{\oplus}$, and a period of 3 days; and Kepler $10 \mathrm{c}$, a 17 $m_{\oplus}$ planet with a radius of $2.35 R_{\oplus}$ in a 45 day period. ${ }^{5}$ HARPS-N continues a very active observing program with continuing follow-up of exoplanet candidates from the Kepler and K2 missions as well as the ongoing RPS program.

\section{ASTRO-COMB}

To enable sub $10 \mathrm{~cm} / \mathrm{s}$ calibration of the HARPS-N spectrograph, required for detecting terrestrial exoplanets in the habitable zone of Sun-like stars, we have deployed a laser frequency comb (LFC) based calibrator, ${ }^{6-13}$ referred to as an astro-comb. ${ }^{14,15}$ This green astro-comb produces over 7000 narrow lines $\left(<10^{-6} \mathrm{~nm}\right.$ width $)$ spaced by $16 \mathrm{GHz}(0.02 \mathrm{~nm}$ at $550 \mathrm{~nm})$ with wavelengths stabilized to the Global Positioning System (GPS) and with flat power from 500 to $620 \mathrm{~nm}$ (Fig. 1). The narrow lines are used to calibrate the spectrograph and measure its line profile. ${ }^{16}$

The astro-comb is based on a $1 \mathrm{GHz}$ repetition rate Ti:sapphire laser whose near infrared light is shifted to visible wavelengths using a photonic crystal fiber (PCF) and then filtered using a pair of broadband FabryPerot cavities to match the resolution of the HARPS-N spectrograph (Fig. 2). To validate the wavelength calibration accuracy of our astro-comb, before deploying it to the TNG, we performed a series of measurements of unwanted LFC line suppression testing the efficacy of the Fabry-Perot filter cavities to suppress undesired comb teeth using three complementary techniques: heterodyne detection, measurement of the FPC transmission profile around $532 \mathrm{~nm}$ combined with phase error and finesse measurements across the full astro-comb spectrum using a commercial optical spectrum analyzer, and direct measurements of the LFC comb line relative powers using a Fourier transform spectrometer (FTS) capable of resolving the $1 \mathrm{GHz}$ source comb spectral lines. These measurements confirmed that the undesired lines of the LFC are suppressed by greater than $40 \mathrm{~dB}$ providing 10 $\mathrm{cm} / \mathrm{s}$ accuracy for the astro-comb when calibrating HARPS-N. ${ }^{17}$

By injecting comb calibration light into both the science and calibration fibers of HARPS-N we find that the short term stability of HARPS-N is measured to be less than $2 \mathrm{~cm} / \mathrm{s}$ and the long-term drift of the spectrograph is approximately $10 \mathrm{~cm} / \mathrm{s}$ per day. ${ }^{15}$ We have also measured the line profile of HARPS-N. We fit a convolution of the fiber profile (a skewed half circle), optical aberrations (16 orders of Hermite-Gaussian modes), and the CCD pixel response (top hat function) to these observed astro-comb spectra. The results indicate an instrument line profile consistent with the design of HARPS-N: a line profile varying slowly in width and shape across each order of the spectrograph. On the blue end of each order the PSF is narrower and skewed to the blue relative to pixel center and on the red end the PSF is broader and skewed to the red relative to the pixel center. ${ }^{15}$ The astro-comb has been partially automated with future work planned to turn the astro-comb into a fully automated, turn-key instrument.

\section{SOLAR TELESCOPE DESIGN}

The small solar telescope, mounted on the outside of the TNG telescope dome, is a simple design optimized to uniformly capture light from the full solar disk. The telescope consists of a $200 \mathrm{~mm}$ focal length, 3 inch aperture lens focusing light into a 2 inch diameter integrating sphere whose output is fiber-coupled via a 300 micron 


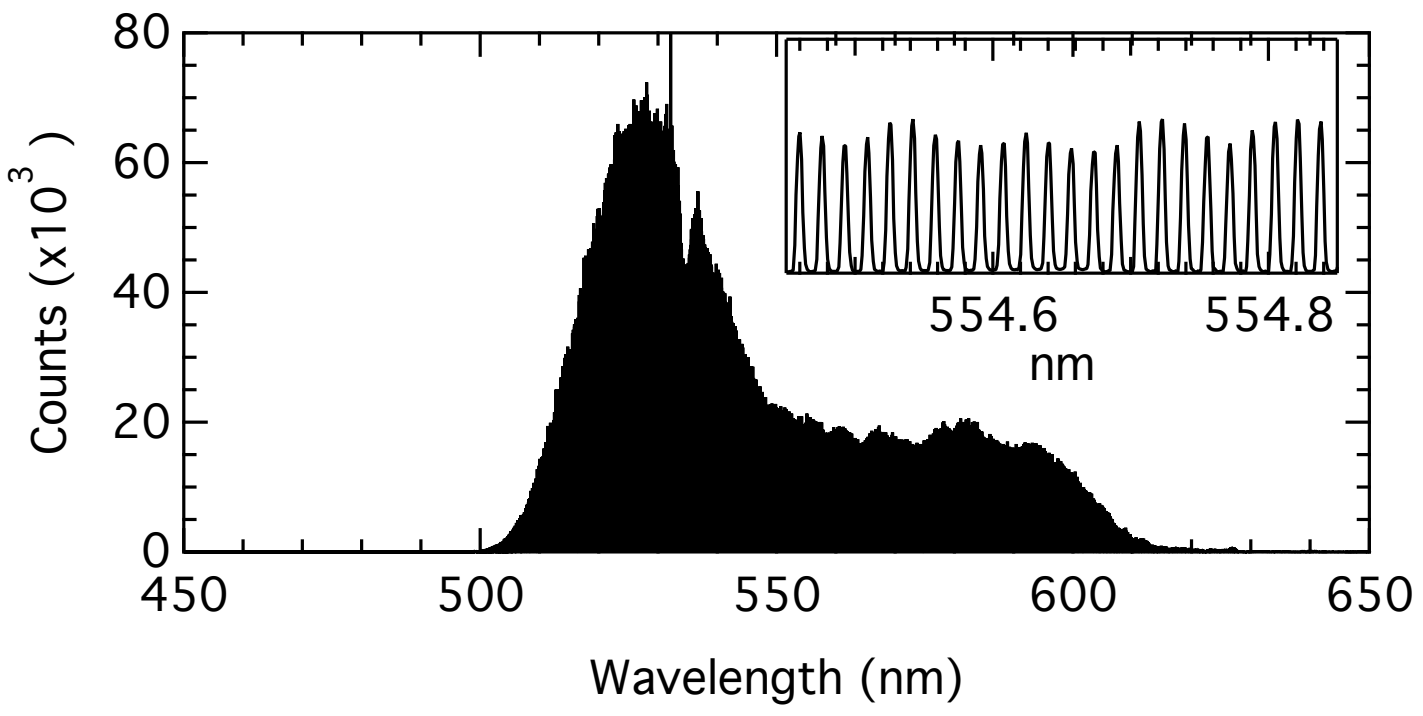

Figure 1. Sample astro-comb spectrum. The calibration spectrum covers 500 to $620 \mathrm{~nm}$ and is flat to within $8 \mathrm{~dB}$. The spectrum contains over 7000 lines spaced by $16 \mathrm{GHz}(0.02 \mathrm{~nm}$ at $550 \mathrm{~nm})$. Inset: $0.4 \mathrm{~nm}$ of comb spectrum showing individual calibration lines. Note that the intrinsic width of these lines is less than 0.001 resolution elements and thus shows the line profile of the spectrograph.

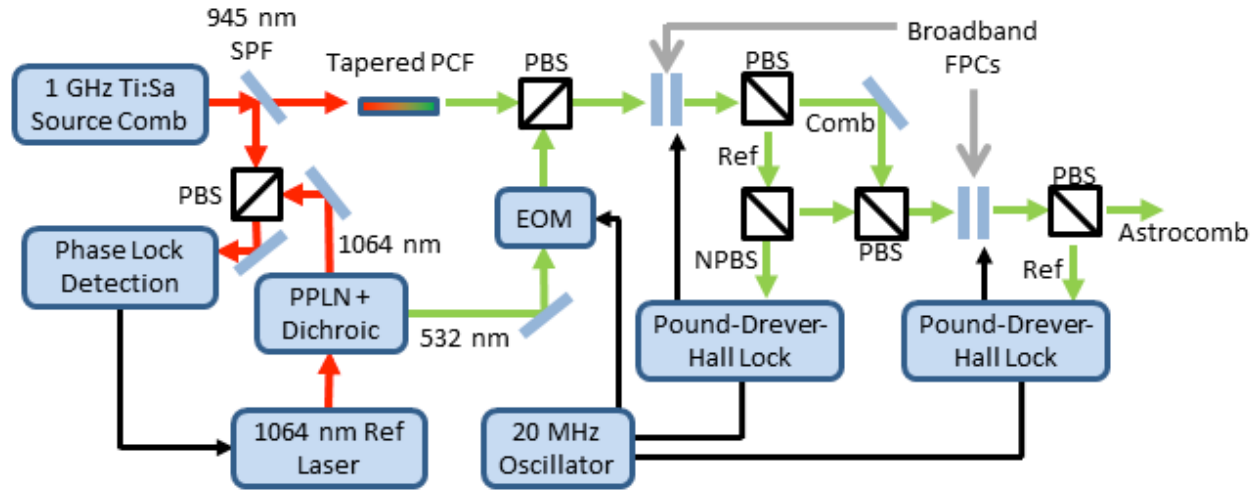

Figure 2. Block diagram of the astro-comb. A $1 \mathrm{GHz}$, femtosecond Ti:sapphire (Ti:Sa) frequency comb (source comb) is split by a $945 \mathrm{~nm}$ short pass filter (SPF) into IR and visible components. The IR component is used to phase lock a $1064 \mathrm{Nd}$ :YAG reference laser to the source comb. The visible component is coupled into a custom tapered PCF for spectral shifting to green wavelengths. The reference laser is frequency doubled with a periodically poled lithium niobate (PPLN) crystal, given $20 \mathrm{MHz}$ sidebands via an electro-optical modulator (EOM), and combined with the comb light via a polarizing beam splitter (PBS). The comb and reference laser light is then coupled into two Fabry-Prot cavities (FPC) that accept every 16th comb line and reject other lines. After each cavity the reference laser and the comb light are separated and the reference laser light is used to lock the cavities using the Pound-Drever-Hall method. (NPBS: non-polarizing beam splitter.) 

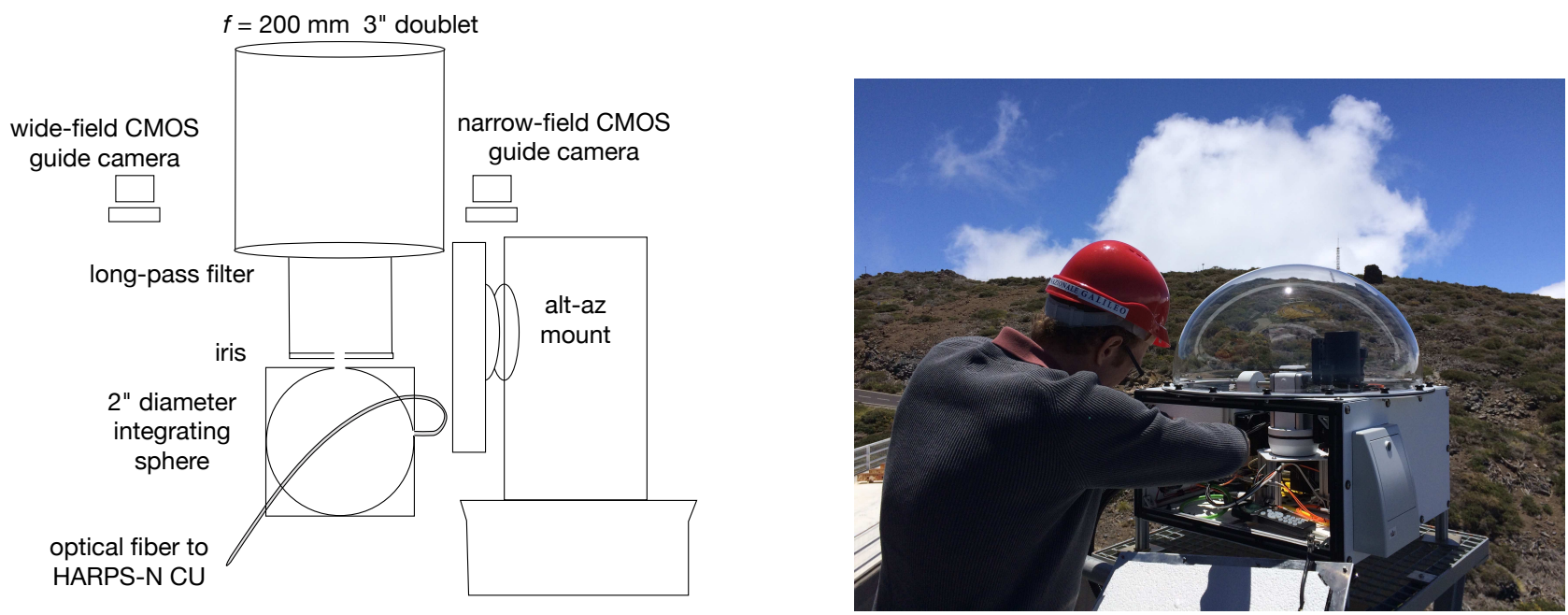

Figure 3. Left: Solar telescope schematic: a $200 \mathrm{~mm}$ focal length, 3 inch aperture lens focuses light into an integrating sphere whose output is fiber-coupled to the calibration unit of the HARPS-N spectrograph. The telescope tracks the Sun using an amateur alt-az mount. Guide cameras built from commercial CMOS cameras provide images of the Sun and provide feedback to keep the telescope centered on the solar image. Right: Alex Glenday completes installation of the solar telescope at the TNG.

multi-mode optical fiber to the calibration unit (CU) of the HARPS-N spectrograph (Fig. 3). The telescope tracks the Sun using an amateur alt-az mount. Guide cameras built from commercial CMOS cameras provide images of the Sun and provide feedback to keep the telescope centered on the solar image.

To allow for robust, unattended operation, the telescope is placed under a fixed, acrylic dome. All signals are sent from the control computer located inside the TNG to the solar telescope via USB-over-fiber, as well as from the solar telescope to the HARPS-N CU via fiber to minimize electrical connections between the solar telescope and the TNG. Key features that allow unattended operation of the solar telescope are: (i) siting in an undisturbed, quiet location; (ii) ability to acquire the Sun each morning via the guide camera; and (iii) automation of data acquisition.

\section{TESTS OF SOLAR TELESCOPE}

We performed tests both in the lab and under operation with the solar telescope feeding sun light to HARPS-N to insure that the design functioned as desired. In the laboratory we studied the integrating sphere to check that telescope throughput was independent of input angle to allow comparisons with SDO images accurate at the 10 $\mathrm{cm} / \mathrm{s}$ level. With a xenon lamp acting as a solar simulator, we translated a collimated beam across the integrating sphere input and studied the throughput as a function of input angle. We found that for angles up to two solar radii, the throughput agreed to better than $10^{-4}$. By removing the output coupler, we also compared images of the solar simulator with first the left half of the image blocked in a re-imaged plane before the integrating sphere and then the right half. Inside the area coupling to the output optical fiber, the output patterns agree to within fractional intensities of $10^{-4}$ insuring that any differences in coupling across the input facet of the fiber will not lead to variations in the output coupling enabling $10 \mathrm{~cm} / \mathrm{s}$ comparisons with SDO images. We also performed tests "on sky" in which while observing the Sun with the solar telescope and HARPS-N we repeatedly offset the guiding of the solar telescope by one solar radius both in azimuth and elevation. We found residual shifts approaching $30 \mathrm{~cm} / \mathrm{s}$ for 1 solar radius offsets of the solar image in the guide camera. To achieve $10 \mathrm{~cm} / \mathrm{s}$ operation thus requires very modest guiding of $20 \%$ of a solar diameter.

\section{OBSERVING WITH THE SOLAR TELESCOPE}

We acquire high signal-to-noise ratio spectra with 20 second integration times after coupling light from the solar telescope into the HARPS-N CU. For each exposure, we realize SNR $\approx 300$ in each resolution element across the 


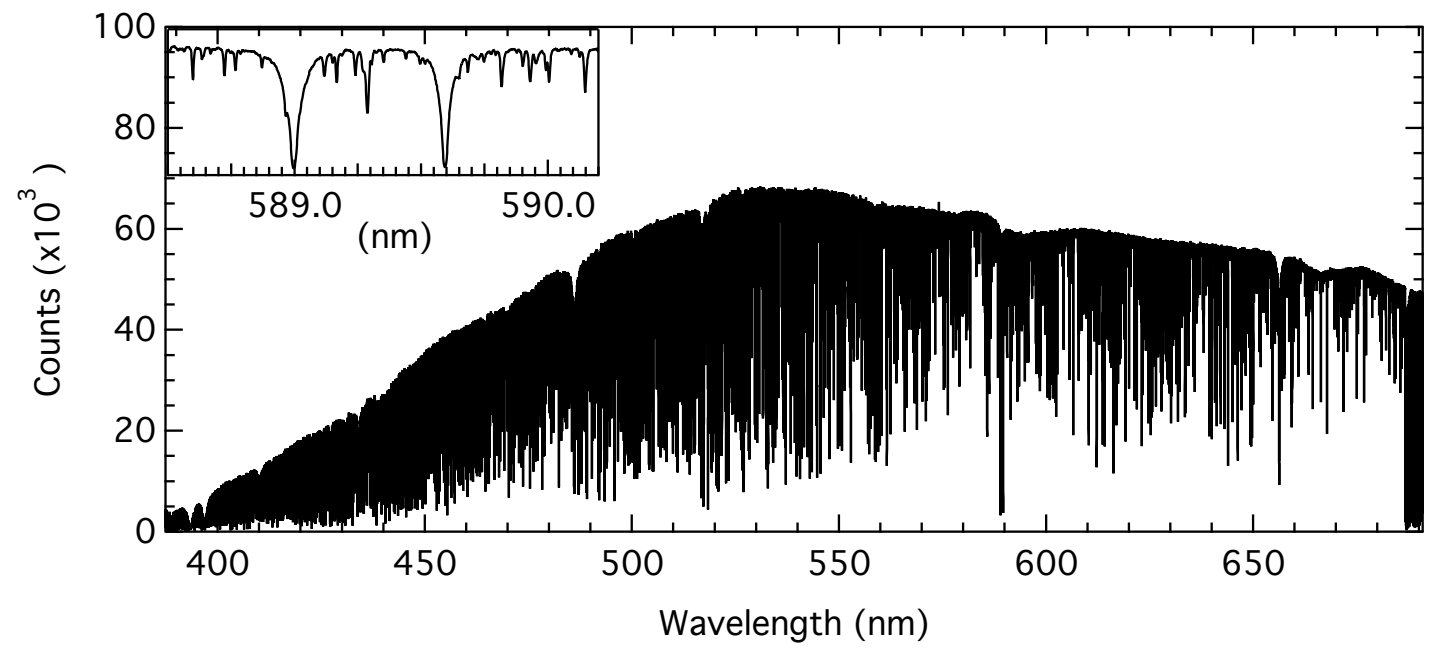

Figure 4. Sample solar spectrum acquired with the solar telescope feeding HARPS-N. While blue wavelengths are attenuated by the additional fiber run from the solar telescope to the HARPS-N calibration unit, the full spectral range of HARPS-N is observed. The inset shows a small piece of the solar spectrum around the sodium doublet.
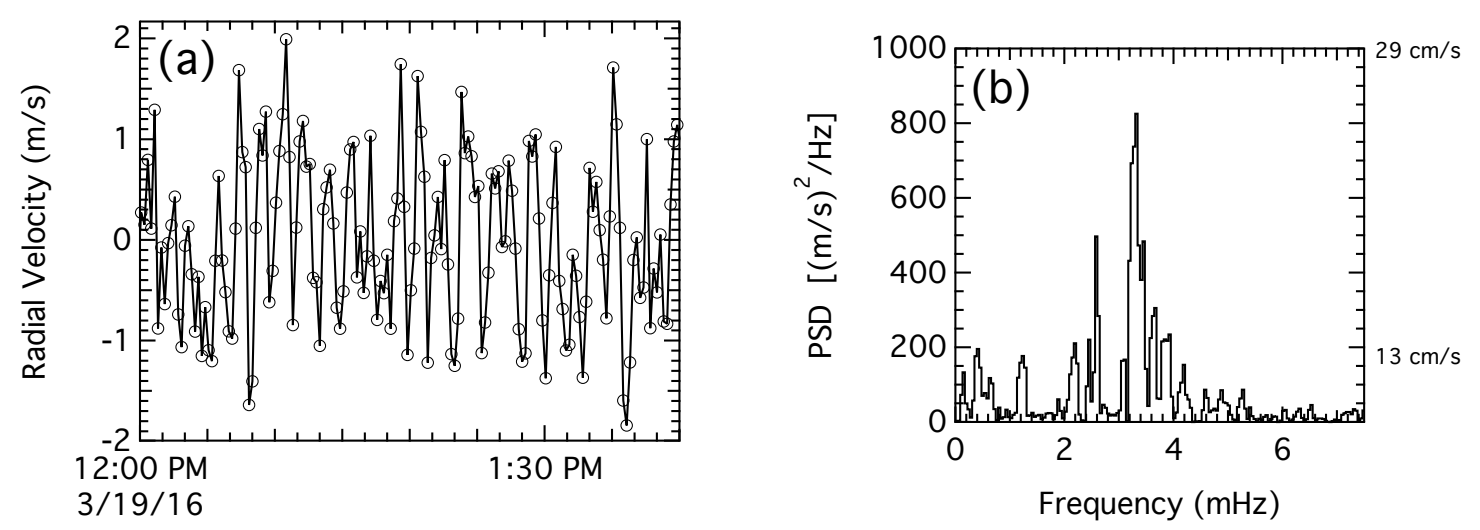

Time (UT)

Figure 5. (a) Solar radial velocities from two hours of 20 second solar exposures showing oscillations from solar p-modes (5 minute oscillations) and (b) Power Spectral Density (PSD) of the Fourier Transform of the time domain RVs. Note that the bandwidth of each bin is $83.5 \mu \mathrm{Hz}$.

green orders of the observed spectrum. During such exposures, we use the astro-comb for simultaneous reference, monitoring changes in the wavelength calibration of HARPS-N. Figure 4 shows an example spectrum. We then use either the standard HARPS-N pipeline or an independent pipeline to reduce solar spectra and extract solar radial velocities, referenced either to the solar system barycenter or directly to the JPL Horizons ephemeris containing the motions of all solar systems bodies. We find uncertainties in individual points of $30-50 \mathrm{~cm} / \mathrm{s}$ within $30 \%$ of photon noise.

Acquiring repeated 20 second exposures over several hours allows us to observe the solar p-modes (Fig. 5a). We find good agreement with short-term variations in radial velocities as extracted from the BiSON network of telescopes. ${ }^{18,19}$ After performing a Fourier Transform upon the extracted RVs, the $3 \mathrm{mHz}$ period of the p-modes is evident. Averaging approximately 1 hour of measured RVs acquired from 80 spectra (including detector read time) leads to uncertainties in the measured RV below $10 \mathrm{~cm} / \mathrm{s}$ (see right axis of Fig. 5b). 
(a)

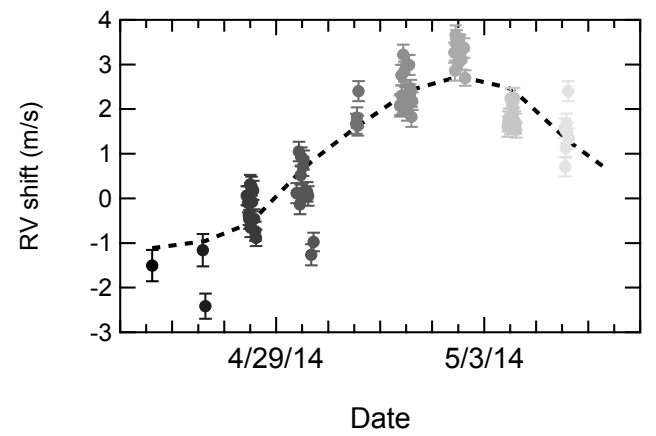

(b)

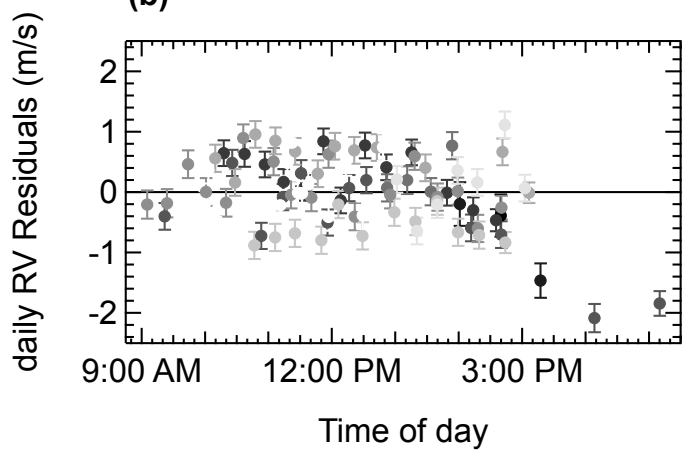

Figure 6. (a) Solar radial velocities over 9 days with expected values from JPL Horizons ephemeris subtracted. Each point consists of the weighted average of three 5 -minute exposures. The fit (dashed line) uses the $F-F^{\prime}$ technique ${ }^{20,21}$ relying upon full disk visible wavelength photometry of the Sun. (b) Residuals after subtracting daily means showing near photon shot-noise RMS variations at the $30 \mathrm{~cm} / \mathrm{s}$ level. Error bars in (a) and (b) are derived from weighted averages of each echelle order of the HARPS-N data.

To match more typical observations of stellar targets, we observe the Sun with HARPS-N with five minute exposures. Since the solar telescope provides sufficient light to optimally fill CCD pixels in 20 seconds, we introduce neutral density filters in the CU to attenuate the light from the Sun by a factor of 15 before it enters the spectrograph, matching the SNR of the 20 second exposures. Figure 6 shows the extracted RVs for 9 days of initial observations with the solar telescope and HARPS-N performed in 2014. We observe variations in the measured solar RV over this time period of several $\mathrm{m} / \mathrm{s}$ which are well modeled by simple photometric models. $^{20,21}$ We note that after additional observations, we find that this model sometimes accounts well for observed fluctuations in the solar RVs and less well in other observations. As in the twenty second exposures described above, we find uncertainties in the range of $30-50 \mathrm{~cm} / \mathrm{s}$ with the variation due to solar p-modes mostly eliminated via the longer exposures (Fig. 6b).

We are currently observing the Sun every clear day with the solar telescope and HARPS-N. We typically acquire 4 to 6 hours of 5 minute exposures. To date, when scientists from the astro-comb collaboration are not present to operate the astro-comb, we calibrate the spectrograph and monitor drifts with a passive Fabry-Perot cavity $^{22}$ referenced to a thorium emission lamp. Every few months, we operate the astro-comb to provide a cross calibration between the thorium lamp calibration and the astro-comb to study and compensate for longterm drifts in the thorium lamp. Over the span of many months, we observe RV rms scatter from the Sun of approximately $2 \mathrm{~m} / \mathrm{s}$, demonstrating the need for compensation for the effects of solar activity on the measured RV.

\section{COMPARISON WITH SDO IMAGES}

To study the effects of solar activity on RV measured with the solar telescope and HARPS-N, we are comparing the RVs to images acquired by the HMI instrument onboard the Solar Dynamics Observatory. ${ }^{23}$ Since its launch in 2012, SDO has provided continuous observations of the solar surface brightness, velocity, and magnetic fields with image resolution finer than the photospheric granulation pattern via the HMI instrument. This allows us to probe the RV variations of the Sun in unprecedented detail (Fig. 7) using the Fe I $617.3 \mathrm{~nm}$ line. Recent work on comparing solar RVs to HMI images by members of our collaboration ${ }^{24}$ has shown success at the $1 \mathrm{~m} / \mathrm{s}$ level in correcting for solar variations of the observed RV of the asteroid Vesta, by determining the RV variation arising from both the suppression of granular blueshift in magnetized regions and the flux imbalance induced by dark spots and bright faculae. We are currently applying these techniques to RVs from the solar telescope to correct for the effects of activity and enable the $10 \mathrm{~cm} / \mathrm{s}$ sensitivity required to observe the reflex motion of the Sun due to Venus in its 584 day synodic period. 

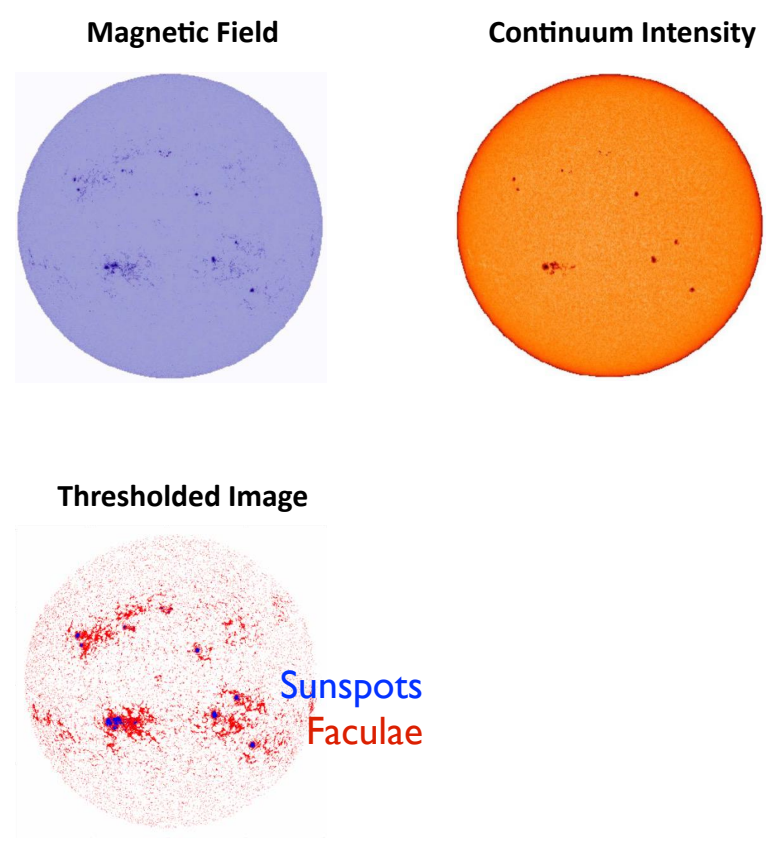

\section{Line-of-Sight Velocity}

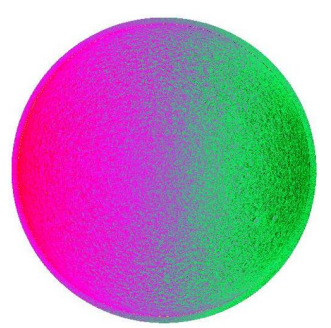

Line-of-sight velocity (Solar Rotation Removed)

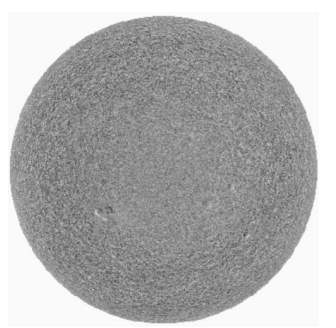

Figure 7. Sample images from the Solar Dynamics Observatory (SDO) HMI instrument which uses narrow-band measurements of the $617.3 \mathrm{~nm}$ Fe I line to extract the radial velocity and magnetic field with a $1^{\prime \prime}$ angular resolution of the Sun. In the top row, we show the magnetogram highlighting magnetic activity, the continuum near the Fe I line, and the radial velocity as observed with the iron line. In the bottom row, we show (left) the magnetogram after thresholding to detect active regions with faculae (red/lighter shade pixels) and sunspots (blue/darker shade pixels) and (right) the velocity after removing solar rotation.

\section{CONCLUSIONS}

Initial tests with the solar telescope on HARPS-N show sub m/s RV precision on short time scales from hours to days and few $\mathrm{m} / \mathrm{s}$ precision on time scales of months likely due to activity. Since late July of 2015 , the solar telescope has been operating every clear day, and such observing will continue over the next several years to collect a data set from which to extract the signature of Venus from solar radial velocities. Success in these observations would demonstrate the capability of HARPS-N using the radial velocity technique to detect terrestrial exoplanets in the habitable zone.

\section{REFERENCES}

[1] Dumusque, X., et al., "HARPS-N observes the Sun as a star," Astrophys. J. 814, L21 (2015).

[2] Cosentino, R., et al., "HARPS-N @ TNG, two year harvesting data: performances and results," Proc. SPIE 9147, Ground-based and Airborne Instrumentation for Astronomy V, 91478C (2014).

[3] Pepe, F., et al., "An Earth-sized planet with an Earth-like density," Nature 503, 377380 (2013).

[4] Motalebi, F., et al., "The HARPS-N Rocky Planet Search I. HD219134b: A transiting rocky planet in a multi-planet system at 6.5 pc from the Sun," Astron. Astrophys. 584, A72 (2015).

[5] Dumusque, X., et al., "The Kepler-10 Planetary System Revisited by HARPS-N: A Hot Rocky World and a Solid Neptune-Mass Planet," Astro. Phys. J. 789, 154 (2014).

[6] Murphy, M. T., et al., "High-precision wavelength calibration of astronomical spectrographs with laser frequency combs," Mon. Not. R. Astron. Soc. 380, 839847 (2007). 
[7] Li, C.-H., et al., "A laser frequency comb that enables radial velocity measurements with a precision of 1 $\mathrm{cm} / \mathrm{s}, "$ Nature 452, 610612 (2008).

[8] Steinmetz, T., et al., "Laser frequency combs for astronomical observations," Science 321, 13351337 (2008).

[9] Braje, D. A., Kirchner, M. S., Osterman, S., Fortier, T., and Diddams, S. A., "Astronomical spectrograph calibration with broad-spectrum frequency combs," Eur. Phys. J. D 48, 5766 (2008).

[10] Quinlan, F., Ycas, G., Osterman, S., and Diddams, S. A., "A 12.5 GHz- spaced optical frequency comb spanning > $400 \mathrm{~nm}$ for near-infrared astronomical spectrograph calibration," Rev. Sci. Instrum. 81, 063105 (2010).

[11] Wilken, T., Lovis, C., Manescau, A., Steinmetz, T., Pasquini, L., Lo Curto, G., Hänsch, T. W., Holzwarth, R., and Udem, T., "High-precision calibration of spectrographs," Mon. Not. R. Astron. Soc. Lett. 405, L16-L20 (2010).

[12] Ycas, G. G., Quinlan, F., Diddams, S. A., Osterman, S., Mahadevan, S., Redman, S., Terrien, R., Ramsey, L., Bender, C. F., Botzer, B., and Sigurdsson, S., "Demonstration of on-sky calibration of astronomical spectra using a $25 \mathrm{GHz}$ near-ir laser frequency comb," Opt. Express 20, 6631-6643 (2012).

[13] Wilken, T., et al., "A spectrograph for exoplanet observations calibrated at the centimetre-per-second level," Nature 485, 611-614 (2012).

[14] Li, C.-H., et al., "Green astro-comb for HARPS-N," Proc. SPIE 8446, Ground-based and Airborne Instrumentation for Astronomy IV, 84468X (2012).

[15] Glenday, A. G., et al., "Operation of a broadband visible-wavelength astro-comb with a high-resolution astrophysical spectrograph," Optica 2 (3), 250-254 (2015).

[16] Phillips, D. F., et al., "Calibration of an astrophysical spectrograph below $1 \mathrm{~m} / \mathrm{s}$ using a laser frequency comb," Opt. Express 20, 13711-13726 (2012).

[17] Glenday, A. G., et al, "High-resolution Fourier transform spectrograph for characterization of echelle spectrograph wavelength calibrators," Proc. SPIE 8446, 844696 (2012).

[18] Hale, S. J., Howe, R., Chaplin, W. J., Davies, G. R., and Elsworth, Y. P., "Performance of the Birmingham Solar-Oscillations Network (BiSON)," Solar Physics 291, 128 (2015).

[19] Davies, G. R., Chaplin, W. J., Elsworth, Y., and Hale, S. J., "BiSON data preparation: a correction for differential extinction and the weighted averaging of contemporaneous data," Mon. Not. R. Astron. Soc. Lett. 441, 3009-3017 (2014).

[20] Meunier, N., Desort, M., and Lagrange, A.-M., "Using the Sun to estimate Earth-like planets detection capabilities, Astron. Astrophys. 512, A39 (2010).

[21] Aigrain, S., Pont, F., and Zucker, S., "A simple method to estimate radial velocity variations due to stellar activity using photometry," Mon. Not. R. Astron. Soc. 419, 3147 (2012).

[22] Wildi, F., Pepe, F., Chazelas, B., Lo Curto, G., and Lovis, C., "A Fabry-Perot calibrator of the HARPS radial velocity spectrograph: performance report," Proc. SPIE 7735, Ground-based and Airborne Instrumentation for Astronomy III, 77354X (2010).

[23] Pesnell, W., Thompson, B., and Chamberlin., P., "Solar Dynamics Observatory (SDO)," Solar Phys. 275, 3-15 (2012).

[24] Haywood, R. D., et al., "The Sun as a planet-host star: proxies from SDO images for HARPS radial-velocity variations," Mon. Not. R. Astron. Soc. Lett. 457, 3637-3651 (2016). 